\title{
A Case for Old-Fashioned Observability, and a Reconstructed Constructive Empiricism
}

\author{
Hasok Chang ${ }^{\dagger}$
}

\begin{abstract}
I develop a concept of observability that pertains to qualities rather than objects: A quality is observable if it can be registered by human sensation (possibly with the aid of instruments) without involving optional interpretations. This concept supports a better description of observations in science and everyday life than the object-based observability concepts presupposing causal information-transfer from the object to the observer. It also allows a rehabilitation of the traditional empiricist distinction between observations and their interpretations, but without a presumption that observations are infallible. Using this concept of observability, I also propose a re-formulation of constructive empiricism that is easier to defend against realist attacks, while open to reasonable realist intuitions.
\end{abstract}

1. Introduction. The concept of observability has been a subject of philosophical debate for many years, especially in the context of the recent arguments concerning scientific realism. The aim of this paper is to formulate a notion of observability as pertaining to qualities rather than objects. This constitutes a challenge to the recently popular philosophical accounts of observation that treat it as a process of causal informationtransfer from the observed object to the observer (e.g., Shapere 1982, Kosso 1988). In some ways, the concept of observability I propose signals a return to a more traditional empiricism, although this return is made with a full awareness of the difficulties created by the theory-ladenness of observation.

Why would I want to make such a move back to an apparently antiquated position? There are two main reasons. First, the object-based concept of observability is inadequate for dealing with many instances of

$\dagger$ To contact the author, please write to: Department of Science and Technology Studies, University College London, London WC1E 6BT, United Kingdom; e-mail: h.chang@ucl.ac.uk.

Philosophy of Science, 72 (December 2005) pp. 876-887. 0031-8248/2005/7205-0019\$10.00 Copyright 2005 by the Philosophy of Science Association. All rights reserved. 
what we normally regard as observation; the quality-based concept can do much better. Second, my re-definition of observability can serve to reorient the realism debate in a more productive direction. More specifically, I believe that the object-based definition of observability, which van Fraassen shares with many realists, introduces an unwarranted realist bias, which should be removed. Using the quality-based concept of observability, I propose a reconstructed constructive empiricism that is more resolutely empiricist, yet more open to realist arguments and intuitions in specific situations.

2. Motivations for the Quality-Based Concept of Observability. Traditional empiricists were agreed that the starting point of our knowledge was the reports of sensation, hence qualities rather than objects. Carnap $(1995,225)$ defined 'observable' as a term applying to "such properties as 'blue', 'hard', 'hot'," which are "directly perceived by the senses." More recently, however, the general consensus seems to be that observability is an attribute of objects ${ }^{1}$ rather than of qualities. This object-focus is shared by philosophers right across the epistemological spectrum from Shapere to van Fraassen, although there are some dissenters, like Humphreys (2004, 23-25), who argues for "property realism." Shapere $(1982,492)$ calls "directly observable" any "entity" from which information is transmitted "directly, i.e., without interference" to an appropriate receptor. Van Fraassen $(1980,15)$ says that "the term 'observable' classifies putative entities," and by "entities" he means "things and events." The case of Kosso $(1988,451)$ is telling: Although he discusses observability as pertaining to specific qualities, he can only conceive of them as belonging to objects - that is, as properties. ${ }^{3}$ I wish to argue against this object-focus in the concept of observability.

Many observations do not have physical objects attached to them. In order to make that point, I need to engage in some phenomenology of what we normally take to be observations, both in everyday life and in

1. There is no complete agreement about the ontological terminology here. Van Fraassen includes 'events' as a type of 'entities', and seems to equate 'things' with 'objects'. I will use the terms 'entity', 'object', and 'thing' interchangeably in this paper, and understand them as denoting physical beings located in space and time. But I am open to any sensible terminological modifications.

2. Rynasiewicz (1984, 190-192) has also raised some objections to the object-focus, arguing that observability should be regarded as an attribute of abstract situations rather than concrete objects and events.

3. Kosso's full definition is as follows: "The ordered pair $\langle$ object $\mathrm{x}$, property $\mathrm{P}\rangle$ is observable to the extent that there can be an interaction (or chain of interactions) between $\mathrm{x}$ and an observing apparatus such that the information that $\mathrm{x}$ is $\mathrm{P}$ is transmitted to the apparatus and eventually to a human scientist." 
scientific research. ${ }^{4}$ Consider first the nature of physical measurements, which are the best that modern science can boast in the category of 'observations'. Measurements are of quantities, not physical objects. When we measure the pitch of a sound, for example, we may or may not know the source of the sound; we may just be dealing with disturbances in the air, like wind, not straightforwardly caused by an easily identifiable object. Or picture the moment when William Herschel discovered infrared radiation in 1800: He sticks a thermometer into the dark space beyond the red end of the solar spectrum, and measures a certain increase in the thermometer reading. What object is he observing with the thermometer? Whose temperature is he taking? There is no clear answer. When we make measurements of physical constants, such as the gravitational constant or Planck's constant, there are surely physical objects involved in the experiment, but what is measured is not a property belonging to those objects. Or consider the measurement of time: "It is 2:35 pm now." The "it" in that report is just a grammatical filler (of a kind that does not exist in all languages) masking the fact that there is no particular object being described in the sentence.

Everyday experience also shows the routine occurrence of object-less observations. I walk into the room and observe: "It's stuffy in here," or "It stinks." What is it that I am observing? A stinking object, perhaps a rotten egg? What if there is no egg, but someone just synthesized some hydrogen sulphide $\left(\mathrm{H}_{2} \mathrm{~S}\right)$, which can be done in a test tube or in the intestines? In that case, should we say I am only observing the hydrogen sulphide molecules? But then, should we not always say we are just observing the molecules, even when they are given off by an egg? It is not clear how we should conceptualize the situation in terms of objects. But we can simply say: "It smells." Or suppose I pick up a prism, pass sunlight through it and throw a part of the resulting spectrum on my left eye. All I can report is "it's green"; green it certainly is, but I cannot say what 'it' is. The sun is not green, nor is the prism. We cannot escape by saying that the photons hitting my eyes are green, as they are certainly not green in the way grass is green; the 'green' photons, as far as we know, are the generators of my sensation of greenness, but they are not themselves green. Something very much like this happens when we observe a rainbow; van Fraassen $(2001,162,156 f f)$ admits that "rainbows are not objects, events or processes." The point is that in these and similar cases the observation consists in registering a quality, and that the observations are perfectly meaningful and coherent without any knowledge or presumption of an

4. Some of these cases will not be considered as acts of observation according to the definition of "observation" that I propose later in the paper. 
object to which the quality belongs, or even an object that causes the quality to be present.

If we still want to classify objects as 'observable' or 'unobservable', we can do so as follows. An object is observable if any of its properties are observable; it is unobservable if it has no observable properties. But this definition of object-observability is parasitic on the notion of qualityobservability, and keeping that in mind helps us avoid some confusions and unhappy locutions. One thing that becomes immediately obvious is that even an observable entity can have unobservable properties. In fact, it is hard to imagine an observable entity to which our modern science does not attribute all sorts of unobservable properties, such as microstructures. (We will have occasion to come back to this point in the final section.)

Remembering the primacy of qualities in observability also helps us avoid the common mistake of regarding ordinary medium-sized objects as observable and everything else as unobservable just because we can see the former and not the latter. Once we recognize that there are many kinds of qualities that can provide observability, it is very easy to avoid this error, which I will term "ocularism"- the privileging of the sense of vision over all others, which also often results in an unhealthy obsession with size. We need to recognize that a sufficient number of chlorine molecules sprayed in the air are just as observable as a rock that hits my head. Ocularism is an error more often committed in loose speech than in careful philosophical writing, but there are some curious exceptions. For example, Vollmer $(2000,356)$ focuses completely on vision in her discussion of observability, and declares that we should regard as observable any entities that can be observed "on the basis of the same physical principle on which entities that can be seen with the naked eye are observed," which involves the scattering of a wave and the performance of an inverse Fourier transform. ${ }^{5}$ This is naturalism that has lost its plot. If it makes no sense to privilege human sensation, it makes even less sense to privilege one particular mode of human sensation (vision), and then to make a strange generalization to privilege all the interactions that are like human vision. Vollmer $(2000,361,365)$ says that caffeine is an observable entity because we can discern its molecular structure through X-ray crystallography. I say caffeine is observable through the buzz I feel after I ingest it (and indirectly observable through the unimaginable number of people who stay awake at philosophy conferences).

5. Muller (2004, 88, 95) also exhibits clear occularist tendencies, despite sophistication in other respects. 
3. The New Old-Fashioned Definition of Observability and Observation. Building on the considerations given in the last section, I propose the following definition of observability and observation: ${ }^{6}$

A quality is observable if it can be registered by human sensation and the claim of registering that quality is not destroyed by the withdrawal of optional interpretations.

Observation is an act of gathering information by human sensation without optional interpretations.

As hinted in the last section, it is important not to forget the richness of our physical experience. The qualities we can observe include not only what is accessible to all of the 'five senses' (vision, hearing, smell, taste, touch), ${ }^{7}$ but also the sensation of temperature, humidity, tension (pressure, weight, etc.), temporal duration, and a collection of other qualities that can only described as 'feelings' verging on the emotional (pain, suffocation, nausea, sleepiness).

Stipulating that observations should not involve any optional interpretations allows us to revive the old intuition that observations provide 'facts', namely information that we cannot control at will and therefore has to be understood as received from the 'external world'. It is useful to rehabilitate the much-maligned idea that sense-data are incorrigible. That idea surely is groundless if it is taken to mean that sense-data are guaranteed to be true. Better insight comes from sticking literally to the etymology of the term 'incorrigibility' - the impossibility of correction, not truth or infallibility (incorrigible people are often very wrong). Observations are incorrigible not because they are true in some ultimate sense, but because we have no means of changing them at will.

In this vein, we can regard an instrumentally-registered quality as quasiobservable if the use of the instrument is de facto incorrigible, and does not involve any interpretations that are considered optional. In these cases we simply use an instrument, without questioning whether it is operating correctly or analyzing the mechanism by which it works. In other words, these are situations in which we treat an instrument as if it were a sense-

6. In an earlier publication (Chang 2004, 86) I defined observation as "reliable determination from sensation"; I think it was a mistake to tie observation so closely to reliability, though I do hope to work out a notion of reliability which renders that definition of observation fully compatible with the one I am proposing here.

7. Curiously, Teller $(2001,134)$ mentions in a similar vein only three of the five senses: "what will count for us as observable phenomena will have more to do with what we experience as unfolding in real time in our visual, auditory, and perhaps tactile sensory fields." Perhaps the exclusion of smell and taste gives him away as a physicist, not a chemist, at heart. 
organ, or literally an extension of our senses. All this, of course, is not to say that there are no circumstances under which the instrument could not be questioned or itself become an object of investigation. But in those cases the instrument loses its incorrigibility, and hence its status as a provider of direct observations.

There are two ways in which an instrument could be used without involving optional interpretations. First, we may employ an instrument to enhance our sensations, with a total acceptance of the instrumentmediated input as sensation itself. This is what we do when we put on our glasses; once that commitment is made, we do not go around trying to interpret in this way or that way the individual images seen through the glasses. That becomes even more obvious if we consider irreversible acts like eyesight correction surgeries. Teller (2001, 132-133) has argued that observations using stethoscopes and microscopes are also immediate in the same way. Second, instruments can create new observable qualities by providing operational definitions. In such cases, a perceivable property of an instrument is taken as a definitive indicator of an unperceivable quality; the translation between the two is made by a fixed rule, with no room for optional interpretations case-by-case. For example, in an ordinary chemistry lab the length of the mercury column in a standard thermometer is taken as a straightforward indication of temperature. Such operational definitions may apply to qualities that are not perceivable at all (e.g., magnetic fields, observed with a compass). Or they may apply to qualities that are perceivable but not to the degree of precision desired or not in the relevant range of magnitudes (consider, for example, how we rely on thermometers for precision temperature measurements or the measurements of very high and very low temperatures, though temperature in the rough sense is directly perceivable in the medium range).

4. Humanist Empiricism. I must now consider various possible objections to my definitions. The likely objections are very well known, which is another way of saying that we would do well to remember why traditional definitions of observation and observability had been rejected in the first place. By the time I have finished answering these objections, I will have sketched a new brand of empiricism that I will characterize as 'humanist'.

Observation as I construe it will seem to echo too strongly the discredited old notion of sense-data. Perhaps the most devastating objection to sense-data was that no system of knowledge could be built on them. This was a point already raised within the Vienna Circle during its heyday, in the course of the famous "protocol sentence" debate. Even Schlick ([1934] 1979, 382) conceded this problem with observation statements (which he called "affirmations"): "Upon affirmations no logically tenable 
structure can be erected, for they are already gone at the moment building begins. If they stand in time at the outset of the process of knowledge, they are logically of no use." Therefore, he thought that the only clear role to be played by affirmations was in the verification of theories.

In my view, Schlick conceded too much here. The uselessness of sensedata is an illusion created by the logical-positivist background. Of course, if we are only allowed to perform straightforward logical operations on "red here now" and such statements, we would not be able to get anywhere very interesting (even if we allow inductive logic). But building empirical knowledge is a much more complicated process of interpretation. In trying to make sense of "red here now" and "round shape" and "cool hardness" followed by "shape getting larger (as arm flexes)" then "crunchy feeling and tangy sweetness" and then "a dent in the round shape and some of the redness replaced by whiteness" and so on, we construct an apple. Starting from various sensations of hot and cold we construct the concept of temperature as a property of bodies, and refine it into a precise quantitative notion with the help of increasingly sophisticated thermometers. (I have discussed the case of temperature in detail in Chang 2004.) From such interpretations, giving us everyday objects and properties, we proceed to make layers upon layers of complicated interpretations, until we reach dinosaurs, DNA molecules, neutrinos, and the Big Bang. ${ }^{8}$ Although we have, each of us and collectively, come a very long way from dealing with direct observations alone, it makes no sense to deny that observations do provide the starting points and some crucial later ingredients for our evolving interpretations.

At that point another objection arises: It makes no sense to distinguish observations from their interpretations, because observations themselves already embody interpretations. This objection is of course based on the theory-ladenness of observation, and all I can say is that the problem is exaggerated much too often. A lot of the examples pulled out in order to illustrate theory-ladenness concern interpretations of observations, not observations themselves; they only prove that interpretations of observations rely on theoretical assumptions, which is not disputed. This is not to deny that interpretations may enter our experience unconsciously and unintentionally. Even so, they may still be optional (otherwise we would probably not be able to recognize them as interpretations at all). Optional interpretations can be withdrawn or destroyed by reflection. Even Shapere, who insists that we go along with the scientists' claim to "observe" the interior of the sun by detecting solar neutrinos, freely admits that the

8. Bogen and Woodward (1988) give a good sense of how some of this building-up is done in the middle stages, when they describe the identification of phenomena from data. 
layers of interpretation included in what he considers "observation" can and will be peeled away if specific doubts arise about them, until we reach the level of "dot" or "smudge" $(1982,518-519)$.

Polanyi (1958, 55-65) himself argues that many tacit interpretations are not initially given, but acquired as hard-earned skill. What are initially meaningless shocks delivered to the hands of the novice user of a walking stick later become interpreted as distinct physical objects; with further practice, the interpretation may even become completely unconscious. Still, with an appropriate reminder the skilled practitioner is able to recognize the presence of interpretations. ${ }^{9}$ In growing up, most of us do become very expert at the tacit interpretation of our observations in terms of three-dimensional objects, but that is not an indication that such an interpretation cannot logically be separated from the observations themselves. Physicalism is a gigantic program of interpretation, not an ineliminable ingredient of observation itself. (A corollary of the tacitness of many interpretations is that "experience" can be, and typically is, much richer than pure observation.)

So let us admit the cogency of the rather traditional distinction between observation and its interpretation. But many commentators have challenged the epistemic privileging of human sensation implicit in that distinction. My view is that the human-focus is a problem only if it is taken to mean that human sensation is somehow guaranteed to be certain or correct. I make no such assumptions. There is no necessary correlation between the immediacy of our access to something and its trustworthiness, although immediacy does often create a psychological feeling of certainty ("I saw it with my own eyes," people say, referring to a ghost or a UFO). As mentioned already, the incorrigibility of observations only means that we are stuck with them, not that they are correct. This incorrigibility is admittedly not absolute, either. Just as the reliance on certain instruments can be withdrawn by agreement, so can the credence in some human observers (declared insane), their specific modes of sensation (as colorblind, hard of hearing, etc.), or specific instances of observations (as hallucinations). Still, we are pretty well stuck with taking the testimony of human senses on the whole as a starting point of our empirical knowledge. There is no anthropocentrism here (certainly not "human chauvinism" as Vollmer puts it), but only humanism in the form of a recognition that we cannot, and should not try to, get away from ourselves. If we were to renounce such humanism, what would be the point of subscribing to empiricism at all?

9. This is in line with van Fraassen's response $(2001,158)$ to Teller's above-mentioned argument that observations using microscopes are immediate. 
5. Reconstructing Constructive Empiricism. In this final section, I wish to comment on the implications of humanist empiricism on the realism debate, particularly regarding van Fraassen's constructive empiricism. The adoption of the quality-based observability concept shields constructive empiricism from some standard realist objections; however, it also necessitates a significant revision in the notion of empirical adequacy and its axiological implications.

Van Fraassen's object-based definition of observability is a trap that continually drags him toward realism. As McMullin (2003) points out, van Fraassen puts his scientific anti-realism in the midst of a broader realism, and it is difficult to police the boundary between the two. Part of the great admiration that van Fraassen inspires is due to the brilliance with which he has managed to escape from this trap. But there is little glory in winning a battle that never was necessary. Adopting the qualitybased definition of observability that I propose can altogether eliminate the difficulty, of which I will emphasize two aspects.

The first aspect is that if we say that observable entities are known to be real, then it becomes difficult to resist the presumption that mere unobservability could not render other things unreal, because it is difficult to see why there would be a meaningful ontological distinction correlated with observability. An emblematic case would be the undeniable reality of the microscopic objects that we presumably get when we smash up the macroscopic objects that we regard as real. This sort of objection was fully anticipated by van Fraassen (1980, 13-19), referring to Grover Maxwell's arguments already published in 1962. Van Fraassen's solution is to emphasize that he is talking about epistemology, not ontology $(1980,19)$ : "[E]ven if observability has nothing to do with existence . . . it may still have much to do with the proper epistemic attitude to science." But we can dispense with the difficulty completely by insisting that observability really has nothing at all to do with the existence of objects, rather than trying to hold on to the idea that observable things must exist. All entities belong in the realm of interpretations, rather than observations. (We do not have thing-perceivers in our sensory apparatus.) Between the observations of qualities and the thing-interpretations of the observations, there is no Maxwell-type continuum. ${ }^{10}$

10. Another difficulty that is dissolved by the adoption of my quality-based concept of observability is that we can only judge the observability of entities by relying on what scientific theories say about those putative entities and their interactions with other putative entities, as van Fraassen freely admits (1980, 56-59). I think van Fraassen's hermeneutic indulgence of this theory-dependence is sound, but again, it is unnecessary. The difficulty simply dissolves if we adopt a quality-based concept of observability. The observability of qualities is judged directly by our ability or inability to perceive them, not by what scientific theories tell us about what we ought to be 
Secondly, there is a hidden realism in van Fraassen's concept of empirical adequacy, and the quality-based concept of observability can eliminate it. Van Fraassen's "preliminary explication" $(1980,12)$ is that "a theory is empirically adequate exactly if what it says about the observable things and events in this world, is true." But if a theory is entirely true about all the "observable things and events" it treats, then it must be true about all of their unobservable properties as well, which is surely far more than a good empiricist should want to commit to. This opens up constructive empiricism to the kind of objection raised by Friedman (1982, 278): since medium-sized "observable" objects are made up of elementary particles, "if I assert that observable objects exist, I have also asserted that certain complicated systems of elementary particles exist." This objection can certainly be countered with some effort (see, e.g., Muller 2004, $82 f f$.), but the effort is unnecessary because the objection simply dissolves if we restrict empirical adequacy to cover only observable qualities.

If what we mean to say is that empirical adequacy is being true about the observable properties of observable entities, ${ }^{11}$ then it is less troublesome and simpler to say that an empirically adequate theory fits observations. I propose to term this modified notion of empirical adequacy "observational adequacy," to avoid confusion with van Fraassen's original concept. Observational adequacy does not concern unobservable qualities, and on the other hand it applies to object-less observations. This way the separation of empirical adequacy from truth becomes cleaner, and much more tenable. Observational adequacy would amount to truth only for theories about entities that have no unobservable properties; logic does not rule out the existence of such entities, but I cannot imagine any in actual science.

Where does all of this leave us in the realism debate? The quality-based notion of observability allows us to remove an important source of unwarranted bias toward realism. The humanist distinction between observation and its interpretation strengthens van Fraassen's position because it emphasizes the 'constructive' in constructive empiricism. Interpretations are constructed, not discovered; they need to fit with observations, but they cannot be proven by observations. As there is always room for choice in the construction, any given interpretation is optional. Therefore all interpretations are corrigible, including those involving entities that are

able to perceive. This judgment of observability is independent from any interpretive understanding of it that we may strive to achieve. We need not torment ourselves with questions like "Can we really go and see the moons of Jupiter?" or "Can we shrink ourselves to the size of a paramecium (and if so, would we be able to see tiny things)?"

11. That is what van Fraassen $(1980,12)$ seems to want when he is speaking "a little more precisely" about empirical adequacy: an empirically adequate theory "has at least one model that all the actual phenomena fit inside." 
assigned some observable properties (which is to say, observable entities). Since all physical objects are part of such interpretations, their reality needs to be argued for in each case. As van Fraassen $(2001,156)$ himself reminds us, we know that some tempting physical-thing interpretations do not succeed: "In some sense we do represent shadows, moving spots of light, reflections, mirages, and rainbows as things. But the phenomena themselves refuse to let us maintain this representation." Only those particular interpretations that are more successful can be accepted as parts of our "observable" reality. But we could be incorrect about the unobservable aspects of observable objects, even when those objects are elements of an observationally adequate interpretation. Therefore even less can be taken for granted for realism than van Fraassen allows.

On the other hand, quality-based observability does not categorically rule out realism regarding objects, either. If the realism question is about whether we can have an absolute proof of the existence of objects and the truth of our theories about them, then the answer is no. But that is not very interesting. I think it is better to focus the realism debate on the question of which of our interpretations of observation we can trust, ${ }^{12}$ and to which degree. This fiduciary question of realism does not have a general answer, and it can only be decided by arguments which take into account the details of each case, or each type of case. One plausible idea is that the synergy of different modes of observation can provide strong grounds for trust; for instance, Hacking $(1983,189)$ cites Berkeley's argument that we can build our trusted three-dimensional representation of the everyday world only by synthesizing vision and tactile sense. But generally there seem to be no inherent limits on what kind of interpretations may be shown to be reasonably trustworthy, so the fiduciary question of realism remains open. Therefore, in order to strengthen van Fraassen's position in the way I suggest, we must let go of one of the chief attractions of his constructive empiricism, namely the clear and general distinction it provides between the kind of knowledge that we may reasonably strive to achieve in science (knowledge about observable entities) and the kind of knowledge that we ought not to demand because it is not within our reach (knowledge about unobservable entities). In other words, as long as the fiduciary question of realism has no simple answer, van Fraassen's answer to the axiological question (concerning the aims of science) cannot be stabilized, either. And this, I believe, is as far as

12. "Trust" is a notion that I will leave without a sharp definition in this paper. It possibly implies a range of things, from the assumption of truth to the reliance for providing explanation and understanding. At the least trust would involve a pragmatic confidence in the future reliability of the interpretation, which results in our willingness to maintain it. 
"pure" philosophy can take us. The concrete debates to resolve the fiduciary question in each case will verge on the scientific, and that is as it should be.

\section{REFERENCES}

Bogen, James, and James Woodward (1988), "Saving the Phenomena", Philosophical Review 97: $303-352$

Carnap, Rudolf (1995), An Introduction to the Philosophy of Science. Edited by Martin Gardner. New York: Dover.

Chang, Hasok (2004), Inventing Temperature: Measurement and Scientific Progress. New York: Oxford University Press.

Friedman, Michael (1982), "Review of The Scientific Image by Bas C. van Fraassen”, Journal of Philosophy 79: 274-283.

Hacking, Ian (1983), Representing and Intervening. Cambridge: Cambridge University Press. Humphreys, Paul (2004), Extending Ourselves. Oxford: Oxford University Press.

Kosso, Peter (1988), "Dimensions of Observability", British Journal for the Philosophy of Science 39: 449-467.

McMullin, Ernan (2003), "Van Fraassen's Unappreciated Realism”, Philosophy of Science 70: $455-478$.

Muller, F. A. (2004), "Can a Constructive Empiricist Adopt the Concept of Observability?", Philosophy of Science 71: 80-97.

Polanyi, Michael (1958), Personal Knowledge. Chicago: University of Chicago Press.

Rynasiewicz, Robert (1984), "Observability", PSA 1984, vol. 1: 189-201.

Schlick, Moritz [1934] (1979), "On the Foundation of Knowledge", in Philosophical Papers, vol. 2. Dordrecht: Reidel, 370-387.

Shapere, Dudley (1982), "The Concept of Observation in Science and Philosophy", Philosophy of Science 49: 485-525.

Teller, Paul (2001), "Whither Constructive Empiricism?", Philosophical Studies 106: 123 150.

van Fraassen, Bas C. (1980), The Scientific Image. Oxford: Clarendon Press.

(2001), "Constructive Empiricism Now", Philosophical Studies 106: 151-170.

Vollmer, Sara (2000), "Two Kinds of Observation: Why van Fraassen Was Right to Make a Distinction, but Made the Wrong One", Philosophy of Science 67: 355-365. 